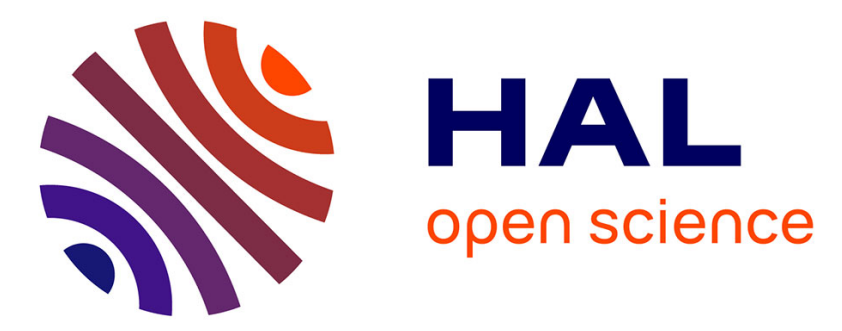

\title{
Automatic extraction of the mitral valve chordae geometry for biomechanical simulation
}

Daryna Panicheva, Pierre-Frédéric Villard, Peter E Hammer, Douglas Perrin, Marie-Odile Berger

\section{- To cite this version:}

Daryna Panicheva, Pierre-Frédéric Villard, Peter E Hammer, Douglas Perrin, Marie-Odile Berger. Automatic extraction of the mitral valve chordae geometry for biomechanical simulation. International Journal of Computer Assisted Radiology and Surgery, 2021, Proceedings of IPCAI 2021, 16 (5), pp.709720. 10.1007/s11548-021-02368-3 . hal-03262496

\section{HAL Id: hal-03262496 https://hal.science/hal-03262496}

Submitted on 16 Jun 2021

HAL is a multi-disciplinary open access archive for the deposit and dissemination of scientific research documents, whether they are published or not. The documents may come from teaching and research institutions in France or abroad, or from public or private research centers.
L'archive ouverte pluridisciplinaire HAL, est destinée au dépôt et à la diffusion de documents scientifiques de niveau recherche, publiés ou non, émanant des établissements d'enseignement et de recherche français ou étrangers, des laboratoires publics ou privés. 


\title{
Automatic extraction of the mitral valve chordae geometry for biomechanical simulation
}

\author{
Daryna Panicheva*,1 • Pierre-Frédéric \\ Villard $^{1,2}$. Peter E. Hammer ${ }^{3}$. Douglas \\ Perrin $^{2,3}$. Marie-Odile Berger ${ }^{1}$
}

\begin{abstract}
Purpose: Mitral valve computational models are widely studied in the literature. They can be used for preoperative planning or anatomical understanding. Manual extraction of the valve geometry on medical images is tedious and requires special training while automatic segmentation is still an open problem.

Methods: We propose here a fully-automatic pipeline to extract the valve chordae architecture compatible with a computational model. First, an initial segmentation is obtained by sub-mesh topology analysis and RANSAC-like model-fitting procedure. Then the chordal structure is optimized with respect to objective functions based on mechanical, anatomical, and image-based considerations.

Results: The approach has been validated on 5 micro CT scans with a graph-based metric and has shown an $87.5 \%$ accuracy rate. The method has also been tested within a structural simulation of the mitral valve closed state.

Conclusion: Our results show that the chordae architecture resulting from our algorithm can give results similar to experienced users while providing an equivalent biomechanical simulation.
\end{abstract}

Keywords Image-based modeling - Mitral valve - Graph-based validation . Biomechanical simulation

\section{Introduction}

The mitral valve is one of the four cardiac valves. It is situated between the left atrium and the left ventricle. The valve is composed of two leaflets and a set of chordae coming from the papillary muscles on the left ventricle and tethered to the leaflets. The chordae maintain the valve competence during systole (ejection) to prevent backflow of blood into the left atrium.

\footnotetext{
${ }^{1}$ Université de Lorraine, CNRS, Inria, LORIA, Nancy, France . ${ }^{2}$ Harvard School of Engineering and Applied Sciences, Cambridge, MA, USA . ${ }^{3}$ Harvard Medical School, Boston, MA, USA · * corresponding author pierrefrederic.villard@loria.fr
} 
Various pathologies could affect the valve efficacy [4]. While surgical procedures are performed for the valve repair, the outcome varies depending on the experience of the surgeon [2]. A computer-based simulation with a valve computational model could improve the surgical treatment either by bringing a better scientific understanding to the clinicians or by providing a tool allowing more accurate preoperative planning.

Different computational models have been studied in the literature. Some include fluid-structure interactions $[8,18,6]$ and others only structural analysis $[16,9,20,7]$. While the former capture the complex influence of the blood flow, the latter are much faster and sufficient in the context of quasi-static behavior at peak systole.

Computational models based on generic valve geometry have been used for pathological cases like annular dilatation [11] or ischaemic dysfunction [5]. However, efficient models for planning could only be obtained with patientspecific geometry as it was demonstrated in [6] by varying the complexity of the chordae architecture. Among the 3D patient-based models in the literature, the study described in [18] uses a mitral valve geometry that is semi-manually segmented from a CT scan acquired on an ovine heart where the mitral valve had been excised and mounted in a in vitro set-up. The same framework has been used for different studies $[10,17]$ to obtain high-quality data with a low level of noise. Putting aside the high precision of the geometry obtained, the use of this acquisition technique is limited as it is not reproducible under clinical conditions. As an alternative, we aim to extract the $3 \mathrm{D}$ valve model from the medical images closer to clinical ones, in order to progress toward a tool for treatment planning.

In recent works based on medical images, where the valve is kept intact in the heart, the chordae are either generated based on the excised measurements, since they are not visible in transesophageal echocardiography [7], or are segmented manually from the micro CT images $[19,20]$. The segmentation procedure is costly in time and requires good anatomical knowledge. There is no method yet to automatically extract the chordae from cardiac medical images. Moreover, once segmented, the chordae have to be attached to the leaflets, which also is performed manually. After the segmentation, the chordae are commonly represented in form of a tree of line segments, including the model proposed in [9].

In this paper, we propose a framework for the mitral valve chordae segmentation from micro CT images with minimum human intervention. The full pipeline is displayed in Fig. 1. With the current state of technology, the image resolution of clinical CT does not allow to properly distinguish the chordae. Micro CT has been chosen with the view that in the future imaging modalities would improve and, at the same time, our method will even gain in robustness. Since the representation of the mitral valve must be suitable for the computational models used for simulation, the framework includes extraction of the chordae geometry in a form of a line segments tree compatible with both mechanical and image-based criteria. We also present a validation procedure for 


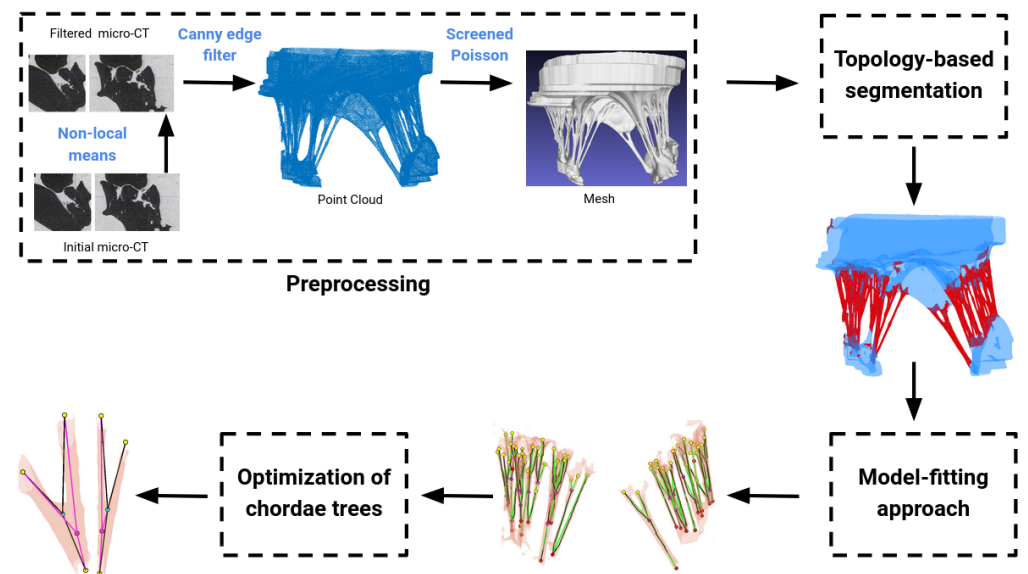

Fig. 1: Chordae extraction pipeline.

the performance evaluation of the proposed method, and we demonstrate its application in the valve closure simulation.

\section{Methods}

\subsection{Segmentation and tree extraction}

The micro CT scans of porcine hearts were acquired post-mortem in the closed valve state simulated with $120 \mathrm{mmHg}$ of air pressure delivered through the aorta (detailed in [19]). As described in [14], chordae are segmented from this data based on their topological characteristics. The contours of the chordae are first extracted with the Canny filter [3] and then the topological shapes are classified according to the number of holes they have (see the chordae extraction pipeline schematically shown in Fig. 1). A chord is composed of quasi-cylindrical parts which are type-2 shapes, and from branching zones, which are equivalent to 3 or more holes shapes, whereas leaflets and ventricle walls are type-0 shapes.

The only parameter involved in this procedure is the maximum radius $R_{\max }$ of the chordae per data, practically it has been measured between 0.6 and $1.3 \mathrm{~mm}$. It is used to estimate the size of sub-meshes extracted to define the topological characteristic at each point. Same parameter $R_{\max }$ will be later applied in sections 2.2 and 3.1. The mesh resulting from this coarse segmentation procedure [14] is used as the starting point for the subsequent geometry extraction.

As chordae are commonly modeled as trees of linear segments in mechanical models like [9], we use a model-based method to extract their geometry. From

1 All the figures are better seen in PDF format. 

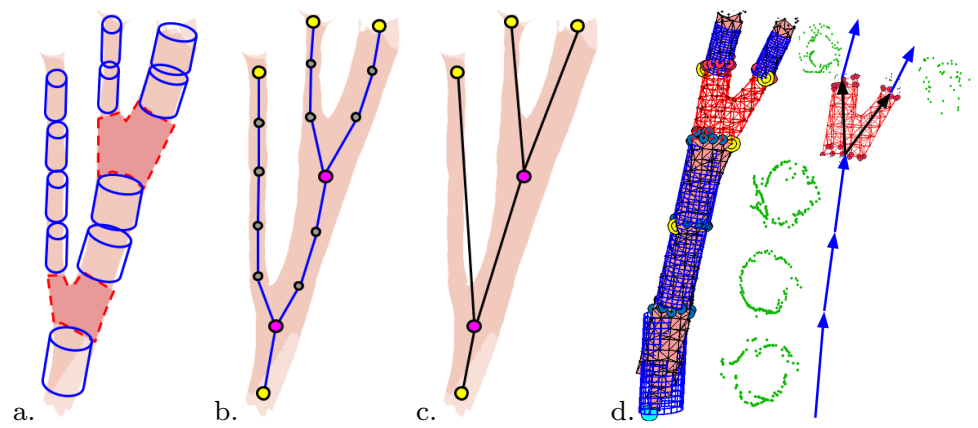

Fig. 2: Model-fitting approach for the chordae geometry extraction: a. Fitted cylinders (in blue) and branching zones (in red). b. Connected cylinder centerlines. c. Resulting tree of line segments after the omission of intermediate points. Terminal points are in yellow, branching points in magenta, intermediate points in gray. d. Example of the constructed chord (data 1). Fitted cylinders are in blue. The initial point of the first sub-mesh is in cyan, initial points of subsequent sub-meshes are in yellow. Black arrows correspond to the connected centroids of the branching zone mesh edges. Green points are the sub-mesh points projected on the fitted cylinder bases ${ }^{1}$.

this point we use chord to refer to the whole tree structure originating from a single starting point at a papillary muscle. Type- 2 shapes of a chord can be geometrically approximated with cylinders with elliptical section. The cylindrical model is fitted locally to the type- 2 shapes with an iterative RANSAC-like approach (MLESAC) that was adapted to our problem. More precisely, we defined an elliptic cylinder model and an acceptance criterion based on the distance of the points to the ellipse after their projection on the cylinder base. This model-based approach with cylinder detection has proven more robust than centerline extraction or skeletonization methods, which usually require a watertight mesh and can be strongly disturbed by non-tubular parts of the mesh that belong to leaflets and papillary muscles.

Starting from the lowest data point the chord is constructed based on the mesh connectivity and the orientation of the previously fitted cylinder as displayed in Fig. 2d. The type-3 shapes (branching zones, shown in red in Fig. 2d.) are used to define the branching directions (shown in black in Fig. 2d.) by connecting the centroids of the sub-mesh edges. To minimize the false detections in the areas close to the leaflet, we additionally check that the points projected on the cylinder base are distributed uniformly (see green points in Fig. 2d.).

The resulting set of connected cylinders and branching zones (schematically illustrated in blue and red in Fig. 2a.) can then be simplified into a set of connected cylinder centerlines and branching points as shown in Fig. 2b. By a branching point we mean a point connected to three or more segments. 
Afterwards, only terminal and branching points are kept, resulting in a tree of line segments (see an example in Fig. 2c.)

The images in the available dataset may have artifacts such as blood clots around the chordae. This causes the presence of type- 4 and more shapes resulting in non-detection of such areas. Consequently, gaps between the segments belonging to the same chord may appear. To avoid it, the cylinder centerlines are connected provided that the angle between their axes is lower than a threshold fixed to $15^{\circ}$. The threshold value has been set based on tests performed on one image and validated on the rest of the images in the dataset.

\subsection{Chordae optimization}

Even if the representation of the chord as a tree of segments is coherent with the image, it may not be appropriate for the mechanical simulation. Indeed, connection of locally fitted cylinders centerlines may create slack in the chordae which leads to the leaflet bulging during the simulation as schematically illustrated in Fig. 3a. This is mainly due to incorrect localization of branching points. Another difficulty is that abnormal topology may appear in the segmented tree. The experimental studies conducted in [10] have shown that the mitral valve chordae branchings should follow the bifurcation pattern, whereas in our procedure more than three segments may start from one branching point. We therefore propose to refine the segmented tree in such a way that (i) its topology is consistent with anatomic knowledge (ii) slack is minimized and (iii) the tree is as close as possible to the chordae centerlines. Unfortunately, no single solution exists that simultaneously optimizes each of theses objectives, thus a trade-off should be defined. We choose to prioritize the respecting of the topology with a tree containing only bifurcations and adopt a lexicographic method [12], where the slack reduction is considered to be more important than proximity to centerlines. The reason for this choice is that image-based optimization results in well centered segments, but still with some slack. The slack chordae are not compatible with the computational model, since our simulation corresponds to the stable state with pressure applied to the leaflets, where chordae are supposed to be in tension. These loose chordae may cause unexpected valve behavior.

Let us first introduce some notation. Each tree is represented by a set of $n$ branching nodes denoted $b=\left\{b_{1}, . . b_{i}, \ldots b_{n}\right\}$. The set of $m$ segments of the tree is denoted $s=\left\{s_{1}(b), \ldots, s_{j}(b), \ldots s_{m}(b)\right\}$. In the following, we first modify the topology of the trees that contain not only bifurcations to make it compatible with anatomical knowledge. Then, given this corrected topology, the set of nodes $b$ is optimized to get a tree that fits the image while minimizing the slack.

Topology optimization. At the chordal trees extraction step, some branching zones are too large and enclose several bifurcations being located close one to another. This results in distorted topology in which a branching point is an origin for three or more chordae (see Fig. 4, red). Given such a node with 


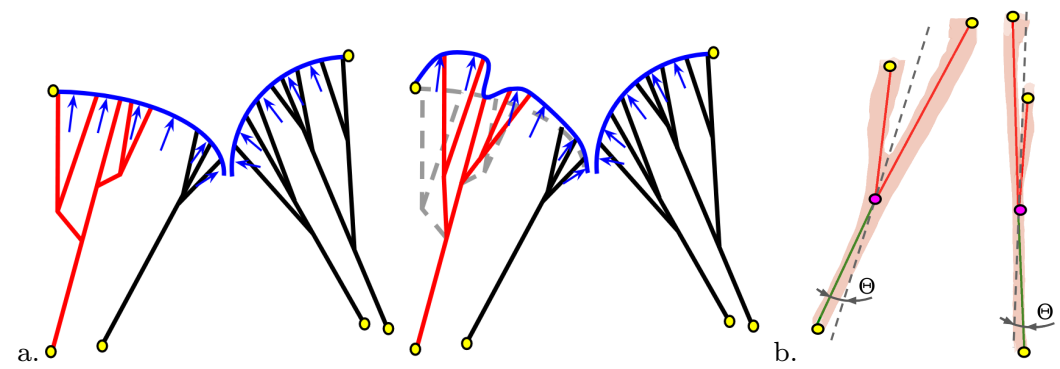

Fig. 3: a. Effect of the chordae slack (slack chordae are in red, chordae with no slack in black) on the leaflet bulging (in blue) during the simulation. b. Angle constraints illustration on a chord shown in two orthogonal views: $\theta$ is the angle between the input segment (in green) and output segments (in red) bisector (in dashed gray), the terminal points are in yellow, the branching node is in magenta. The chordae are in pink.
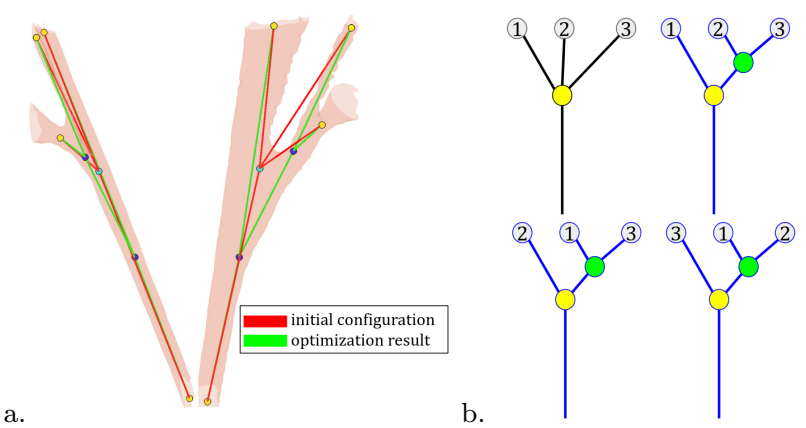

Fig. 4: a. Example of topology optimization results (data 2). The chordae are in pink. b. List of all the three possible binary trees (in blue) corresponding to the tree with initially false topology (in black).

$N_{e}$ output segments, we generate all the $N_{e}-1$ binary trees equivalent to the initial tree where new nodes are the duplicates of the initial one as shown in Fig. 4.b. We perform the optimization for each topology hypothesis. The best configuration is chosen as the one giving the lowest image-driven cost $C_{i m}$.

Once the topology is corrected and the tree has only bifurcation nodes, the position of these new nodes is optimized based on the image criterion described below.

Optimization of the nodes. Two objective functions are defined, one $C_{\text {straight }}$ for quantifying the straightness of the tree and avoiding slack, the other $C_{i m}$ for quantifying the agreement with the image. Given a node of the tree $b_{i}$, we define the straightness of $b_{i}$ as the angle $\theta\left(b_{i}\right)$ between the input segment and the bisector of the two output segments (Fig. 3b.): the smaller this angle is, the more straight the branch is. The global straightness for a 


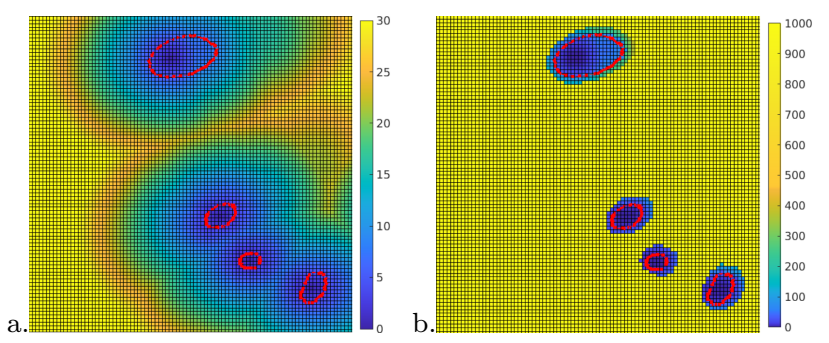

Fig. 5: a. 2D slice of the distance map D. b. 2D slice of the map corresponding to the cost $C_{i m}$. The chordae limits are in red.

given set of branching nodes $b$ is defined as:

$$
C_{\text {straight }}(b)=\sum_{i=1}^{n} \theta\left(b_{i}\right) .
$$

We then determine the set of branching nodes $b$ that minimizes the cost function $C_{\text {straight }}$ :

$$
\min _{b} C_{\text {straight }}(b) .
$$

The image-driven objective function $C_{i m}$ is based on a distance map $D$ indicating how far a chordae segment point $p_{x, y, z}(x, y, z$ are the coordinates) is located from the centerline of the tree (Fig. 5.a). However in order to more heavily penalize points which are outside the chordae volume $V$, we use a greater power of $D$ in the volume $V_{b}$ obtained by dilation of the volume $V$ with a sphere of a size equal to $0.2 \cdot R_{\max }$. Outside $V_{b}$, we use a higher penalization (Fig. 5.b):

$$
C_{i m}\left(p_{x, y, z}\right)= \begin{cases}D\left(p_{x, y, z}\right)^{2} & \text { if } p_{x, y, z} \in V \\ D\left(p_{x, y, z}\right)^{2.5} & \text { if } p_{x, y, z} \in V_{b} . \\ R_{\max }^{3} & \text { otherwise }\end{cases}
$$

As the objective functions are highly non linear, continuous minimization is not appropriate and we instead adopt a discrete approach where a finite number of possible positions (see in blue in Fig. 6a.) is considered around each bifurcation point. In order to define this space, points enclosed by the submesh of the branching zone (determined by the base and branching segments) are extracted. Afterward, the size of the space is reduced by sub-sampling with a cubic grid and taking the centroids of the grid elements.

Two minimal values $C_{\text {straight }}^{0}$ and $C_{i m}^{0}$ are found by exhaustive search while checking all the combinations of possible bifurcation node positions (blue points in Fig. 6a.). Each time a local minimum of the straightness is reached, the value of $C_{i m}$ is evaluated. If this value is less than $C_{i m}^{0}$, then $C_{\text {straight }}^{0}$ and $C_{i m}^{0}$ are updated. We adopt here a multi-scale procedure. It consists in performing the optimization first with coarse discretization for the bifurcation node positions (an example of the result is shown in green in Fig. 6b.) and then with refined discretization (an example of the result is in magenta in 


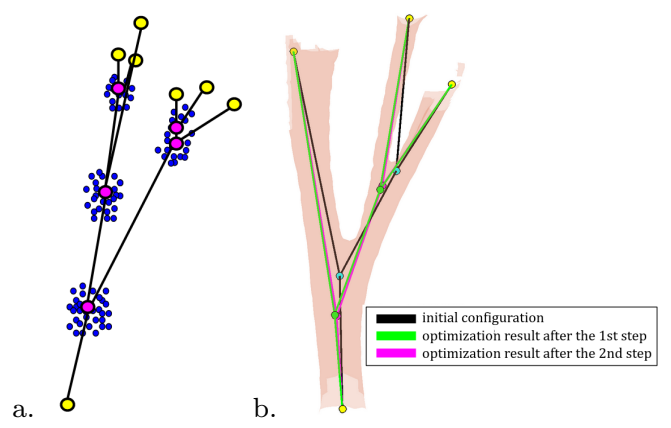

Fig. 6: a. Example of a set of possible positions (in blue) for the branching nodes of the chord (in magenta). The terminal points are in yellow. b. Results of the two steps of the nodes optimization procedure (data2). The chord is in pink.

Fig. 6b). The improvements of both steps are visible comparing to the initial configuration (in black in Fig. 6b): the solution is more straightened and it is closer to the chordae centerlines.

The execution time of the optimization procedure depends on the chordae complexity. Correction of the most frequent case of faulty topology (three segments starting at one point) takes on average 1.8 seconds. The optimization of the position of the nodes takes around 5 seconds per chord with 25 candidate points (in blue in Fig. 6a) per branching node on average. The experiments were performed on an Intel Xeon $3.50 \mathrm{GHz}$ workstation using the Matlab environment.

\section{Results and application}

\subsection{Validation}

The classical approach in image segmentation validation is the comparison of the obtained results with a ground truth $(\mathrm{GT})$. In our case, the results of the chordae extraction are not the labeled images but the geometrical representation of the chordae. Therefore, an equivalent GT chordal structure was obtained via manual segmentation. First, the chordae were delineated by an expert using the 3D Slicer software which resulted in a set of meshes (see Fig. 7 a.). Afterwards, chord by chord, terminal and bifurcation points were manually chosen resulting in a chordal tree (in black in Fig. 7b.). The bifurcation points were chosen by the expert aware of the mechanical application.

The manual segmentation is affected by the expert experience and the variability in the chordal shapes. It may result in significant differences in nodal positions, while the chordae topology is less subject to differences.

The obtained chordal trees can be seen as directed graphs. A metric based on the graph matching can be then applied to compare manually and auto- 


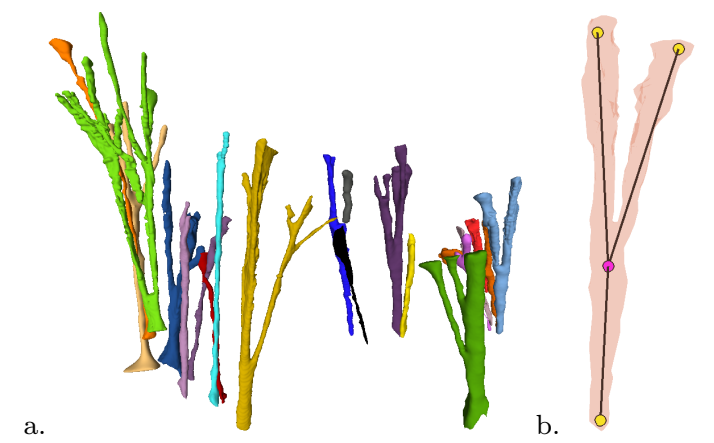

Fig. 7: GT processing: a. Chordae segmented in 3D Slicer (a color per chord, data1). b. Example of the extracted chordal tree (in black) from the segmented surface (in pink). The terminal points are in yellow, the bifurcation point is in magenta.

matically segmented chordal structures. We use a common graph matching method graph edit distance (GED). The value of GED indicates the level of proximity of two graphs. It is calculated as the sum of the costs of the modification operations required to align the two graphs. The operations include substitution, insertion and deletion of vertices and edges. We use the GED algorithm proposed in [1] adapting it to our problem.

The cost of insertion and deletion operations for both vertices and edges is constant and fixed to 1 for simplicity. In order to define the substitution cost for the vertices of two given graphs $G_{1}$ and $G_{2}$ the possibility of matching the $i^{t h}$ vertex of the graph $G_{1}\left(i \in\left[1, n_{1}\right]\right)$ with $j^{\text {th }}$ vertex of the graph $G_{2}(j \in$ $\left.\left[1, n_{2}\right]\right)$ is first determined: if the alignment is possible the substitution cost is 0 , otherwise it is 1 . In our case, vertices are 3D points, so the Euclidean distance is chosen as matching criterion. For the distance threshold value, we have to take into account possible imprecision in the location of the tree terminal nodes. This is illustrated in Fig. 8a. where the displacement of the bifurcation node (orange to blue) is more significant for the larger chord (on the left). Therefore, the alignment of vertices is based on threshold fixed to $\frac{1}{4} \cdot S$, where $S$ is the bounding box diagonal of the chordal tree (see in Fig. 8a.). This allows to take chordae size into account and to be more permissive for the longer chordae where the optimization procedure may induce larger displacements, while not accepting the correspondences between distant and thus not possibly matching vertices.

To define similarity of edges both spatial and orientation proximity are considered. The spatial proximity of two edges $D_{e s}$ is measured by the mean of mutually dropped perpendiculars from the middle points. The angular difference between the edges directions indicates the orientation proximity. In our procedure the $k^{t h}$ edge of the graph $G_{1}\left(k \in\left[1, m_{1}\right]\right)$ can be matched with $l^{t h}$ edge of the graph $G_{2}\left(l \in\left[1, m_{2}\right]\right)$ if the distance $D_{e s}$ between edges is 
a.
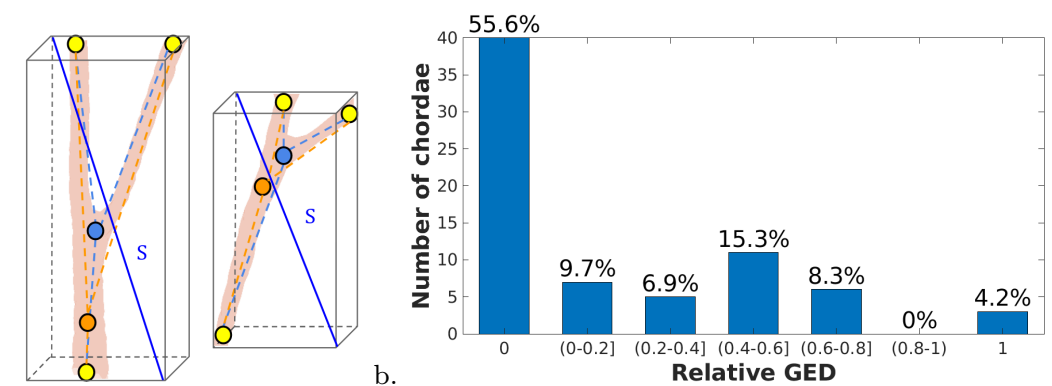

Fig. 8: a. Vertices proximity threshold choice. Initial chord is in blue, chord with displacement is in orange. $S$ is the bounding box diagonal. The terminal tree points are in yellow, chordae are in pink. b. Relative GED value distribution.

less that $\frac{3}{4} \cdot R_{\max }\left(R_{\max }\right.$ is the largest chordae in the data), and the angle difference between edges does not exceed $15^{\circ}$.

Since the cost of the operations is constant for edges and vertices, we can estimate the maximum value of GED for a given chord denoted $G E D_{\max }$. This value corresponds to the complete mismatch of two graphs and is equal to the sum of number of vertices $n_{g t}$ and edges $m_{g t}$ in the GT graph. However, the graphs vary in complexity. This means that those with more branchings have higher $G E D_{\max }$ which complicates the comparison. Thus, we use a relative measure $G E D_{r e l}=G E D / G E D_{\max }$ that indicates the percentage of modifications that can be possibly applied. The complete alignment case corresponds to the $G E D_{\text {rel }}$ equal to 0 since no editions are needed, whereas in the case where $G E D_{\text {rel }}$ is 1 no correspondence is found.

We apply the GED algorithm to the GT chordae trees. For each chordae, the match in the automatically segmented chordal trees set is found as the one requiring the least of modifications, i.e. where the GED is minimum.

The segmentation method was tested on 5 micro CT images. Some chordae are affected by the acquisition artifacts (blood clots, sticking to the ventricle walls, etc.), and thus are ambiguous for the manual segmentation. Such chordae were not taken into account for the validation. The total of 72 chordae were examined. The distribution of the $G E D_{\text {rel }}$ value is shown in Fig. 8b. For 40 chordae $(55.6 \%)$, the actual match was found. Examples of such chordae are shown in Fig. 9. Low values of $G E D_{\text {rel }}$ (less than 0.4) correspond to slight inaccuracies such as shown in Fig. 10a. and b. Higher values of $G E D_{\text {rel }}$ (between 0.4 and 0.6) indicate the absence of significant components in the extracted chordae (see an example in Fig. 10c.). Nevertheless, in all mentioned cases $\left(G E D_{\text {rel }} \leq 0.6\right)$ the GT chordae were matched with the actual correspondences in the segmentation results which is $87.5 \%$ of tested chordae. For such GT chordae the image-driven $C_{i m}$ and straightness costs $C_{\text {straight }}$ were evaluated in order to be compared with the corresponding costs of optimized 


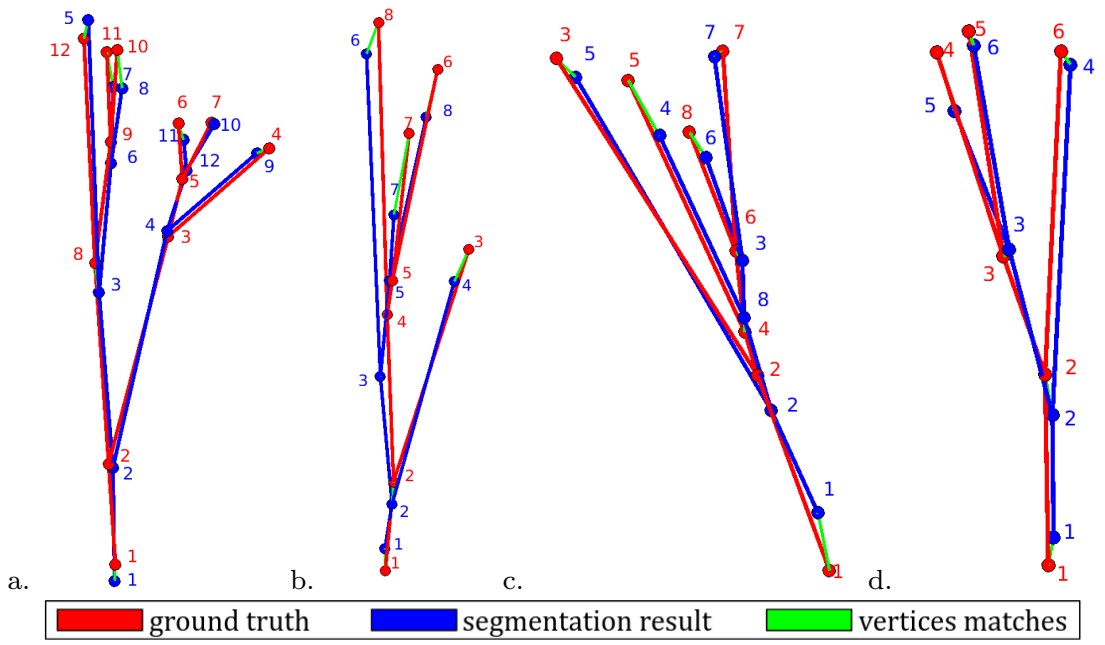

Fig. 9: Examples of total match of automatically segmented chord (in blue) with corresponding GT chord (in red). The matches between vertices are shown in green: a. Data1, b. Data2, c. Data3, d. Data4.

chordae. For all the cases the optimization allows to obtain lower image-driven costs, and for $78.9 \%$ of chordae the straightness term is decreased.

The interval [0.6-1] of $G E D_{\text {rel }}$ corresponds to the false detections (5 in total) and non-detections $\left(G E D_{\text {rel }}\right.$ is 1 for 3 cases). $G E D_{\text {rel }}=1$ errors may obviously impact the realism of the biomechanical simulation. However, such errors are easily discernible by a clinician and can be thus manually corrected. Non-detections are explained by the absence of real correspondences in the segmented chordae resulting either from non-detection at the image contours extraction step ( 7 out of 8 chordae) or due to the false rejection by the modelfitting algorithm (1 out of 8 ). Our experiments showed that incorrectly detected and non-detected chordae mostly concern secondary chordae (see [13] for anatomical details), influence of which on the simulation is restricted.

We note that the proposed metric is designed to distinguish the graphs primarily by the topology. This may lead to the over penalization of the topology inaccuracy. Examples of such cases are shown in Fig. 10d. and e. For both chordae the geometry was globally correctly detected, even if the $G E D_{\text {rel }}$ values are quite high ( 0.7 and 0.57 , respectively). This issue can be addressed by giving more importance to the segments being longer and closer to the tree root. However, it is not straightforward to find the right parameters for such a weighting.

The accuracy of the proposed GED-based metric shows that our automatic pipeline allows to properly extract chordae set architecture.

Another qualitative evaluation that is worth to be mentioned is the fact that another informed user was asked to perform chordae segmentation. After examination, the user acknowledged that the computed solution was better at 


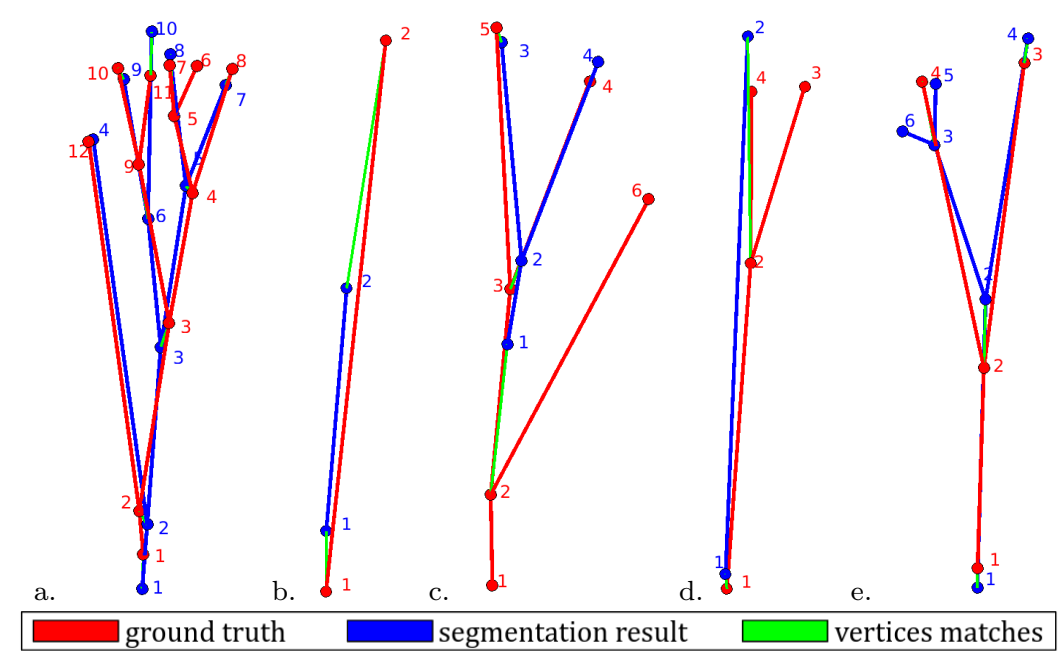

Fig. 10: Examples of correctly matched chordae with inaccuracies: a. Slight inaccuracy $\left(G E D_{r e l}=0.17\right)$, data3. b. More pronounced inaccuracy $\left(G E D_{r e l}\right.$ $=0.33)$, data1. c. Important parts missing $\left(G E D_{\text {rel }}=0.46\right)$, data5. d. Inaccurate topology $\left(G E D_{\text {rel }}=0.7\right)$, data4. e. Inaccurate topology $\left(G E D_{\text {rel }}=\right.$ $0.57)$, data1.

several places of the image than the obtained manual delineation. This shows that the segmentation task is difficult, even for informed users, and that our algorithm delivers a correct segmentation. It can be potentially improved by local manual correction depending on the expertise of the clinician.

Next, we will focus on the application of the chordal set for the biomechanical simulation.

\subsection{Application}

As previously mentioned, the goal of our work is to define a chordae set from a $3 \mathrm{D}$ medical image in such a way that it can be used in biomechanical simulations for clinical procedures. Since the major goal is to ensure valve closure at peak systole, our aim is to evaluate the behavior of the leaflet in its closed position when a constant pressure is applied. In particular, we want to check that our segmentation algorithm does not induce leaflet anomalies such as bulging or leaks. Prior to the simulation, the chordae were automatically extended and attached to the leaflets obtained via manual segmentation. The attachment is done considering the chordae orientation and anatomical angle constraints [10]. The validation procedure detailed in section 3.1 allowed to evaluate the algorithm efficiency, however in real clinical conditions the ground truth is not available. Therefore, for the simulation we have taken the full chordae set 


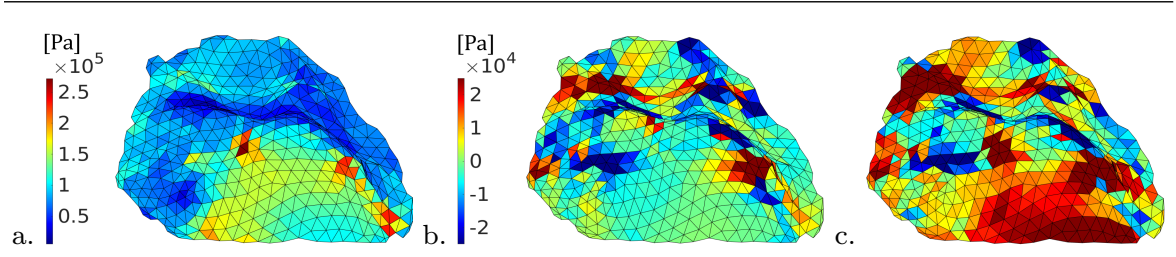

Fig. 11: Simulation results: a. Von Mises stress distribution obtained with GT chordae set. b. Difference between von Mises stresses obtained with GT and optimized chordae set resulting from our method. c. Difference between von Mises stresses obtained with GT chordae set and chordae with enforced slack.

except those for which $G E D_{r e l}=1$. They correspond in practice to abnormal detections which can be easily identified.

The mechanical behavior of the valve is computed as a dynamic system within the framework proposed in [9] where the mass-spring system was replaced by a finite element method (FEM) continuum system. The leaflet is modeled as a triangle mesh (with 591 vertices and 1055 faces), vertices are pinned around the valve annulus. The external forces applied to the leaflet are the tension forces from the chordae, the uniform blood pressure normal to the leaflet surface and the contact forces. The internal forces are computed taking into account large deformation and with a constitutive law using the second Piola-Kirchhoff stress tensor. More details are given in [15].

Three chordal set configurations have been tested within this framework: (i) resulting from the manual segmentation, (ii) resulting from our complete pipeline and (iii) resulting from our extraction method before the optimization (section 2.2) with the chordae slack slightly enforced. The slack configuration was added since it is difficult to compare the configurations with little differences in the attachment points between manually segmented chordae and the optimized results. Therefore, to study the influence of slack on the simulation outcome, we decided to induce slack into the optimized chordae by uniformly elongating them by $10 \%$ in the computational model.

Fig. 11 shows the stress distribution resulting from the simulation using the three different sets of chordae. The results are quite similar to, and in similar ranges as, those found in the literature. As expected, the difference between von Mises stress comparing to GT is more important for the chordae set with enforced slack (see Fig. 11b. and Fig. 11c.), which is explained by less constraint on the leaflets due to slack presence.

We also compared leaflet profiles in a cut plane (shown in Fig. 12a.) obtained in the simulation with the three chordae sets (see Fig. 12b.). As expected, more bulging is observed with the chordae set with enforced slack comparing to two other chordae sets. The geometries resulting from the simulations with the GT chordae set and with our extraction method are very close and both chordae architectures maintain the leaflet shape with few bulging.

For easier interpretation of the results, we added another metric based on bulging volume. Bulging volume is the volume between the leaflet surface at the 
a.

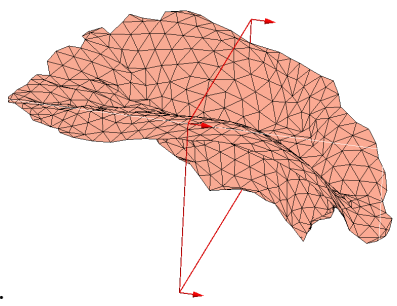

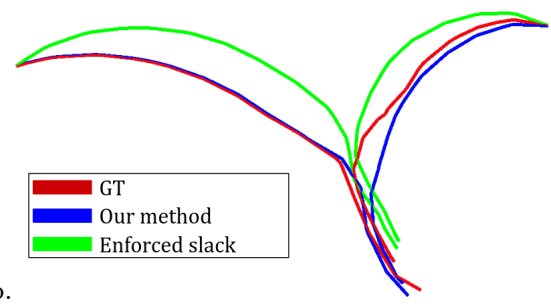

b.

Fig. 12: Leaflet profiles comparison: a. Cut plane in which the leaflet profiles are shown. b. Leaflet profiles obtained with the three sets of chordae.

a.
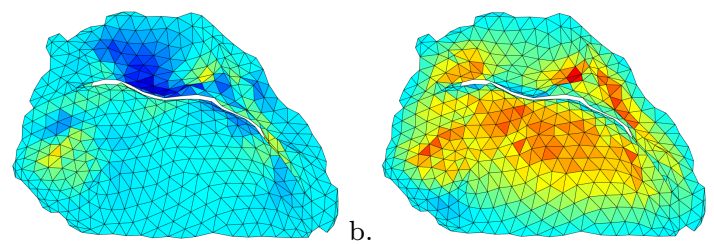

Bulging volume difference, $\left[\mathrm{m}^{3}\right]$

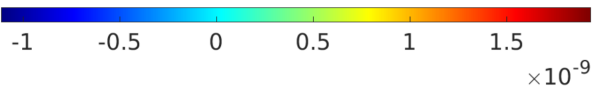

Fig. 13: Map of bulging volume differences: a. Our method minus GT. b. Chordae with enforced slack minus GT.

beginning and at the end of the simulation. Calculated bulging volume values for GT chordae and chordae extracted with the proposed method are the same and are equal to $0.53 \mathrm{~cm}^{3}$. For the slack chordae configuration bulging volume is higher and is equal to $0.92 \mathrm{~cm}^{3}$. The map of bulging volume differences calculated per face between the optimized chordae set and GT chordae is shown in Fig. 13a., while the map of differences between the chordae set with enforced slack and GT is in Fig. 13b. It can be seen that more leaflet bulging is observed for the slack chordae.

Though these results have to be confirmed on a larger cohort, they show that the chordae architecture resulting from our algorithm can give results similar to experienced users while providing an equivalent biomechanical simulation.

The values of the leaflet biomechanical parameters could not be measured in our specific experiments. There are always uncertainties in constitutive law, especially because in our case, the ex vivo tissue properties alter quickly by drying out and become stiffer. Therefore, we decided to study the influence of a stiffer tissue (to mimic the drying out of the leaflets) and a more distensible tissue to confirm that our model has a coherent mechanical behavior. More bulging should be observed when the leaflet is less constrained, and the slack impact should be even more visible with a more distensible leaflet. 


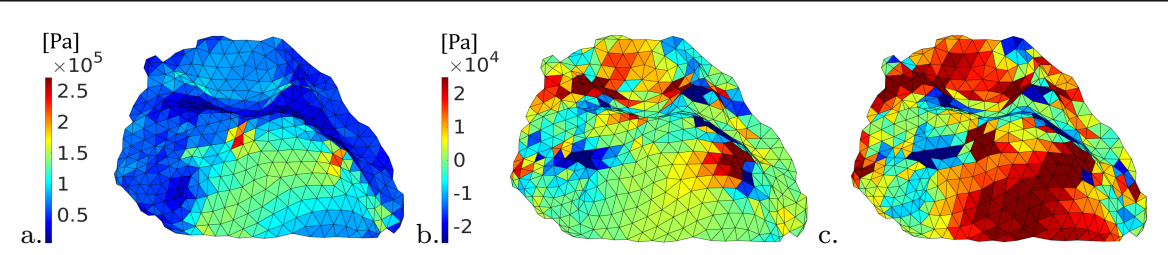

Fig. 14: Simulation results with more distensible leaflet: a. Von Mises stress distribution obtained with GT chordae set. b. Difference between von Mises stresses obtained with GT and optimized chordae sets resulting from our method. c. Difference between von Mises stresses obtained with GT chordae set and chordae with enforced slack.

First, the bulging volumes were calculated for the cases corresponding to the constitutive law variation. For a stiffer leaflet, the bulging volume values obtained with the three chordae sets (GT, optimized chordae set and set with enforced slack) are $0.46 \mathrm{~cm}^{3}, 0.47 \mathrm{~cm}^{3}$ and $0.83 \mathrm{~cm}^{3}$, respectively. Corresponding values for the case of a more distensible leaflet are $0.74 \mathrm{~cm}^{3}$, $0.72 \mathrm{~cm}^{3}$ and $1.1 \mathrm{~cm}^{3}$. As with the initial constitutive law, the values for GT and optimized chordae sets match, and the chordae with enforced slack produce higher bulging. As expected, this study proves the hypothesis that higher leaflet distensibility leads to smaller constraints and hence greater bulging.

Fig. 14 shows stress distribution obtained for a leaflet with increased distensibility. The chordae architecture resulting from our whole pipeline (Fig. 14.b) still leads to small differences with the GT similar to the outcomes obtained with initial constitutive law (Fig. 11.b). Whereas for the set having slack (Fig. 14.c) more significant differences to GT are observed comparing to the leaflet before the distensibility alteration (Fig. 11.c).

\section{Conclusion and discussion}

Extracting accurate chordae architecture of the mitral valve is a complex problem that has no existing solution in the literature. We have proposed in this paper an automatic method for chordae extraction which produces a chordae representation suitable for our computational model. This work is the first step toward patient-based simulations as the proposed approaches allow to decrease manual segmentation bias and thus improve simulation reliability.

We have also designed GED-based metric for the segmentation quality evaluation. The efficiency of the method assessed with the metric has been demonstrated on 5 micro CT scans. Our goal now is to extend it to a larger dataset. Even if the results of the simulation application are mainly qualitative, the leaflet state at peak systole is well reproduced with minimum bulging and in the challenging context of real data.

The limitation of this segmentation method is its relative accuracy in the zones where the chordae meet the leaflet. In these areas the chordae are hardly detectable as they are thin and entangled. We believe that the tethering of the 
chordae on the leaflet is crucial as it deeply impacts the mechanical behavior of the computational model. We thus plan to generate a homogeneous thick structure near the leaflets in order to connect the reliable part of the chordae tree to them.

\section{Declarations}

Conflict of Interest: The authors declare that they have no conflict of interest.

Ethical approval: All applicable international, national, and/or institutional guidelines for the care and use of animals were followed.

Informed consent: This articles does not contain patient data.

\section{References}

1. Abu-Aisheh, Z., Raveaux, R., Ramel, J.Y., Martineau, P.: An Exact Graph Edit Distance Algorithm for Solving Pattern Recognition Problems. In: International Conference on Pattern Recognition Applications and Methods . Lisbon (2015)

2. Badhwar, V., Vemulapalli, S., Mack, M., Gillinov, A., Chikwe, J., Dearani, J., GrauSepulveda, M., Habib, R., Rankin, J., Jacobs, J., McCarthy, P., Bloom, J., Kurlansky, P., Wyler von Ballmoos, M., Thourani, V., Edgerton, J., Vassileva, C., Gammie, J., Shahian, D.: Volume-Outcome Association of Mitral Valve Surgery in the United States. JAMA Cardiology (2020)

3. Canny, J.: A computational approach to edge detection. IEEE Trans. Pattern Anal. Mach. Intell. 8(6), 679-698 (1986)

4. Carpentier, A., Adams, D., Filsoufi, F.: Reconstructive Valve Surgery: From Valve Analysis to Valve Reconstruction. ClinicalKey 2012. Saunders/Elsevier (2010)

5. Cochran, R.P., Kunzelman, K.S.: Effect of papillary muscle position on mitral valve function: Relationship to homografts. Annals of Thoracic Surgery 66(6 SUPPL.) (1998)

6. Feng, L., Qi, N., Gao, H., Sun, W., Vazquez, M., Griffith, B.E., Luo, X.: On the chordae structure and dynamic behaviour of the mitral valve. IMA Journal of Applied Mathematics 83(6), 1066-1091 (2018). DOI 10.1093/imamat/hxy035. URL https://doi.org/10.1093/imamat/hxy035

7. Gaidulis, G., Selmi, M., Zakarkaitè, D., Aidietis, A., Kačianauskas, R.: Modelling and simulation of mitral valve for transapical repair applications. Nonlinear Analysis: Modelling and Control 24(4), 485-502 (2019)

8. Gao, H., Qi, N., Feng, L., Ma, X., Danton, M., Berry, C., Luo, X.: A finite strain nonlinear human mitral valve model with fluid-structure interaction. International journal for numerical methods in biomedical engineering 30(12), 1597-613 (2014)

9. Hammer, P.E., del Nido, P.J., Howe, R.D.: Anisotropic mass-spring method accurately simulates mitral valve closure from image-based models. In: Functional Imaging and Modeling of the Heart, pp. 233-240. Springer Berlin Heidelberg, Berlin, Heidelberg (2011)

10. Khalighi, A., Drach, A., Bloodworth, C., Pierce, E., Yoganathan, A., Gorman, R., Gorman, J., Sacks, M.: Mitral valve chordae tendineae: Topological and geometrical characterization. Annals of biomedical engineering 45(2), 378-393 (2017)

11. Kunzelman, K., Reimink, M., Cochran, R.: Annular dilatation increases stress in the mitral valve and delays coaptation: a finite element computer model. Cardiovascular Surgery 5(4), $427-434$ (1997)

12. Marler, R., Arora, J.: Survey of multi-objective optimization methods for engineering. Structural and Multidisciplinary Optimization 26, 369-395 (2004) 
13. Muresian, H.: The clinical anatomy of the mitral valve. Clinical Anatomy 22(1), 85-98 (2009). DOI https://doi.org/10.1002/ca.20692. URL https://onlinelibrary.wiley.com/doi/abs/10.1002/ca.20692

14. Panicheva, D., Villard, P.F., Berger, M.O.: Toward an automatic segmentation of mitral valve chordae. In: B. Gimi, A. Kro (eds.) SPIE Medical Imaging, vol. 10953, pp. 1095315-1095323. SPIE, San Diego, United States (2019)

15. Panicheva, D., Villard, P.F., Hammer, P., Berger, M.O.: Physically-coherent Extraction of Mitral Valve Chordae. In: International Conference in Computing in Cardiology, vol. 46, p. 4. IEEE, Singapore, Singapore (2019)

16. Prot, V., Haaverstad, R., Skallerud, B.: Finite element analysis of the mitral apparatus: Annulus shape effect and chordal force distribution. Biomechanics and Modeling in Mechanobiology 8(1), 43-55 (2009). DOI 10.1007/s10237-007-0116-8

17. Sacks, M., Sacks, M., Drach, A., Lee, C.H., Khalighi, A., Rego, B., Zhang, W., Ayoub, S., P Yoganathan, A., Gorman, R., Gorman III, J.: On the simulation of mitral valve function in health, disease, and treatment. Journal of Biomechanical Engineering (2019)

18. Toma, M., Jensen, M.Ø., Einstein, D.R., Yoganathan, A.P., Cochran, R.P., Kunzelman, K.S.: Fluid-structure interaction analysis of papillary muscle forces using a comprehensive mitral valve model with $3 \mathrm{~d}$ chordal structure. Annals of Biomedical Engineering 44(4), 942-953 (2016)

19. Villard, P.F., Hammer, P.E., Perrin, D.P., Del Nido, P.J., Howe, R.: Fast Image-Based Mitral Valve Simulation from Individualized Geometry. The International Journal of Medical Robotics and Computer Assisted Surgery 14(2), e1880 (2018)

20. Wang, Q., Sun, W.: Finite element modeling of mitral valve dynamic deformation using patient-specific multi-slices computed tomography scans. Annals of Biomedical Engineering 41(1), 142-153 (2013) 